\title{
IT Innovation: A New Era
}

\author{
Martin Curley \\ Senior Principal Engineer \\ Global Director, Innovation and IT Research \\ Intel Corporation \\ martin.g.curley@intel.com
}

\begin{abstract}
This presentation will discuss the emerging discipline of IT Innovation, a discipline where unique value can be created at the intersection of Information Technology and Innovation. The presentation also shares the structure of an emerging Innovation Capability Maturity Framework and Model while discussing how computing trends such as multicore technology and virtualization will reignite Moore's law and provide a platform for Innovation that is unparalleled in history.
\end{abstract}

IT Innovation is emerging as a new discipline, one which exists at the intersection of two relatively immature disciplines, that of Information Technology and Innovation. Whilst IT is increasingly being recognized as a discipline and the profession is reasonably well developed, it is just in the last couple of years that business schools have started to recognize that Innovation is not just something that happens by luck but perhaps is a process that can be mastered. Driven by the power of Moore's Law IT is a unique innovation resource, it's transformative power and it's ever improving performance/price dynamic making it a very attractive transformation resource. However in parallel IT is also a great resource for helping automate and manage the process of Innovation itself.

For any IT Innovation to be successful, at least six vectors need to be managed successively in parallel

- Vision (Opportunity/Problem)

- IT enabled Solution

- Business Case

- Business Process Change

- Organizational Change

- Customer or Societal Change

To start with somebody has to have a vision of how a particular opportunity can be exploited or how a particular problem can be solved through using information technology or some combination thereof. Typically the vision will be evolved over time. While IT Professionals often think the hardest part of any IT innovation is delivering the IT solution, this often can be the easier part of achieving a new innovation. Since the dotcom crash it is increasingly important and necessary to have a business case associated with any new innovation. The dotcom crash meant that there was a swing from irrational exuberance to irrational pessimism with respect to IT investment. Thankfully there is a 
modicum of rationality returning to IT investment but increasingly a solid business case is a requirement for an innovation to proceed.

The next three vectors are where soft issues come into play and often can be the most problematic part of delivering an IT innovation. Typically an IT Innovation will deliver some form of business process change - in the past typically we saw business process automation but now more often that not we seen business process transformation. This is often accompanies by organizational change, either in the form of new behaviors, new roles or indeed new organization forms. Resource fluidity and organizational and ecosystem dynamics will have a significant influence on the organizational vector. Finally and perhaps most importantly an Innovation is only successful if it is adopted by the customer. Many of today's IT enabled Innovations have societal impact and society's willingness and ability to accept an Innovation is a crucial modulator in the success of an Innovation.

Looking at Innovation as a process there is increasing awareness that Innovation perhaps could be managed as a process. Typically there are three processes involved with an Innovation - cognitive, organizational/logistical and economic. In collaboration with the National University of Ireland, Intel is working to develop an IT Innovation Capability Maturity Framework (ITI CMF) and Model (ITI CMM) to provide organizations with a roadmap and assessment tool to help deliver more value through IT innovation. This framework and model is based on the concept of systemic innovation where synchronous improvements are made to organizational strategy, culture, tools and metrics to help create a virtuous circle of Innovation.

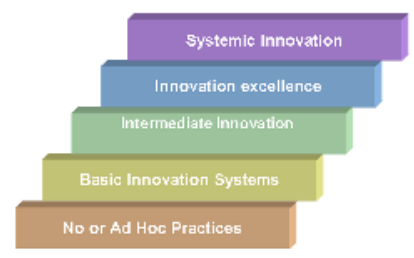

Fig. 1. IT Innovation Capability Maturity Framework (IT CMF). Source: IVI Intel/National University of Ireland.

At the bottom level of the maturity curve the organization has no or ad hoc practices for innovation but as the organization traverses through the curve it steadily increases the maturity and quality of its Innovation Strategy, Culture, Tools and Metrics and ultimately the innovation results. Level 5 maturity, i.e. systemic innovation is when Innovation becomes a way of life and this typically means sustained new profitable growth for a commercial organization.

In parallel with the maturing of IT Innovation as a discipline, IT as a technology continues to evolve. Whilst some were concerned that Moore's Law would peter out as the laws of Physics became barriers for ever increasing frequency, the adoption of a Multicore approach will revitalize and give Moore's law another lease of life. There is a fundamental shift happening in the PC industry as companies move away from increasing the frequency of the CPU to increase performance to the adoption of parallelism as 
the key parameter for delivering better performance. This "right-hand" turn will likely deliver an acceleration of Moore's' Law. The years 2000 to 2004 saw an approximately $3 \mathrm{X}$ improvement in CPU performance but the advent of multicore is projected to deliver a 10X improvement in just the next four years. Multicore is essentially the configuration of multiple logical microprocessors on a single physical die.

Multicore will not just bring better performance, it will also bring better performance per watt, larger throughput and better scalability. As a company Intel is on its third generation of parallelism. Intel's 1st generation of parallel computing was with introduction of purpose-built multi-way servers (DP, MP and SMP). Intel's 2nd generation of parallelism was with Hyper-threading or 2 virtual threads per core, this typically yield up to $30 \%$ performance gains on threaded code and enabled outstanding multi-tasking usage benefits. Intel is now on its 3rd generation of threading with dual core and beyond that combines multicore core with hyper threading for increased parallelism, throughput and versatility. In addition as Virtualization technology diffuses into the marketplace the opportunity expands. Also multicore designs will deliver better performance/watt as easing concerns on the growing power consumption for more and more powerful machines.

As multicore technology develops and as the process of IT Innovation is more managed and ultimately mastered, the future does look bright as a new era of IT Innovation is ushered in.

\section{References}

1. Curley, M. 2004. 'IT Innovation for Competitive Advantage and Adaptiveness", Kluwer, Proceedings IFIP 8.6 Conference. June.

2. Curley, M. 2004. "Managing Information Technology for Business Value", Intel Press. January.

3. Intel internal technical documents, 2006

4. Swanson, Burton E, 2004. "How is an IT Innovation assimilated", Kluwer, Proceedings IFIP 8.6 Conference. June.

5. Pickering, Cindy. 2004. "Using IT Concept Cars to accelerate Innovation", Kluwer, Proceedings IFIP 8.6 Conference. June. 